\title{
Quantum Computation as Gravity
}

\author{
Paweł Caputa ${ }^{1}$ and Javier M. Magan ${ }^{2}$ \\ ${ }^{1}$ Center for Gravitational Physics, Yukawa Institute for Theoretical Physics (YITP), Kyoto University, \\ Kitashirakawa Oiwakecho, Sakyo-ku, Kyoto 606-8502, Japan \\ ${ }^{2}$ Instituto Balseiro, Centro Atomico Bariloche S. C. de Bariloche, Rio Negro, R8402AGP, Argentina
}

(Received 6 September 2018; revised manuscript received 4 March 2019; published 12 June 2019)

\begin{abstract}
We formulate Nielsen's geometric approach to circuit complexity in the context of two-dimensional conformal field theories, where series of conformal transformations are interpreted as "unitary circuits" built from energy-momentum tensor gates. We show that the complexity functional in this setup can be written as the Polyakov action of two-dimensional gravity or, equivalently, as the geometric action on the coadjoint orbits of the Virasoro group. This way, we argue that gravity sets the rules for optimal quantum computation in conformal field theories.
\end{abstract}

DOI: $10.1103 /$ PhysRevLett.122.231302

Introduction and summary.-Information, be it classical or quantum, is intimately related to geometry. This is manifest in the field of computational complexity, which, as shown by Nielsen et al. [1-3], is naturally framed in the language of differential geometry. The main idea is that computational costs can be estimated by distances in the manifold of allowed unitary operations. Therefore, minimizing costs amounts to finding minimal geodesics, and optimal algorithms are given by a free fall through such "complexity geometry." Since Nielsen's approach is fundamentally geometric, it is tempting to expect that it may have relations with general relativity.

On the other hand, the Anti-de Sitter (AdS)/conformal field theories (CFT) correspondence [4] assures us that complexity in CFT, once properly defined, should be encoded geometrically in Anti-de Sitter spacetimes and may, in fact, be crucial to fully understand holography [5]. Up to date, we have two holographic proposals for what "complexity" may be and they usually declare some gravitational notion, such as spacetime volumes or gravity actions, to be related to the state complexity; see Refs. [5-14]. However, it is far from obvious how to define complexity in CFTs. Recent proposals and advances in this direction include Refs. [11,15-27].

The motivation of our work is to bring these developments closer to each other and build bridge(s) between them by developing Nielsen's geometric approach within 2D CFT. In particular, we focus on a subset of quantum symmetry gates (generally discussed in Ref. [27]) that are

Published by the American Physical Society under the terms of the Creative Commons Attribution 4.0 International license. Further distribution of this work must maintain attribution to the author(s) and the published article's title, journal citation, and DOI. Funded by SCOAP ${ }^{3}$. constructed from the energy-momentum tensor and, in 2D CFTs, are fully governed by the Virasoro group.

The universality of our setup allows us to express the complexity action only in terms of the CFT's central charge $c$. We also show that the ambiguity of choosing a metric on Virasoro circuits is a $1 / c$ effect, and at large $c$ the complexity action is given by Polyakov's two-dimensional gravity [28]. This implies that, in our setup within energymomentum gates, gravity governs the rules of optimal computation.

Since the Polyakov and Liouville actions are directly related, our results neatly connect to the recent proposal for path integral complexity [15]. Moreover, it is well known that Polyakov action is equivalent to the coadjoint orbit action of the Virasoro group [29]. This connection between quantum complexity and coadjoint orbit actions is one of the main results of our work and allows for generalizations of our story to coherence groups in arbitrary quantum field theories.

We believe that our approach reintroduces, in a conceptually simple way, important aspects of CFTs which can set the holographic complexity discussion on a firmer ground. Crucially, all our building blocks (the energymomentum tensors and costs) can be defined on both sides of the duality, so holographic complexity proposals should rest on algebraic or geometric relations between them. Last but not least, our considerations certainly open a new unexplored path in the field of quantum complexity in continuous field theories.

Before we begin, we would like to convey a general "take home" message from our study. The concept of circuit complexity comes with a quantum computational package such as universal sets of gates, cost functions etc. that seem hopeless to define in interacting continuous QFTs. On the other hand, motivated by AdS/CFT, we hope that at least in holographic CFTs (strongly interacting 
and with large central charge) complexity can be defined and may play an important role. What we noticed here is that, if we restrict to the "most basic universal gates" in CFTs (energy-momentum tensor is the fundamental object of CFTs) and require large central charge, geometric approach to complexity leads to very universal results. Indeed, not only many of the ambiguities in defining circuit complexity disappear but the universal answer for Nielsen's complexity functional is given by a gravity action (that plays a crucial role in pure gravity sector of $\mathrm{AdS}_{3} / \mathrm{CFT}_{2}$ holography). With our results, we believe that energy momentum and related symmetry gates should be the starting point in understanding field theory complexity also in higher dimensions.

Nielsen complexity and symmetry.-We start by reviewing the geometric approach to complexity based on Refs. [1-3] and [27]. The goal is to estimate computational costs of quantum circuits $U(\tau)$, which in their most generic form are defined as

$$
U(\tau)=\overleftarrow{\mathcal{P}} \exp \left(-i \int_{0}^{\tau} H\left(\tau^{\prime}\right) d \tau^{\prime}\right)
$$

driving us from a given reference state at $\tau=0$ to a particular target state at $\tau$. The Hermitian operators $H(\tau)$ depend on the allowed operations that we have at our disposal (quantum gates) and $\overleftarrow{\mathcal{P}}$ denotes gate ordering (earlier $\tau$ first).

Nielsen's attractive approach to estimate the associate costs is via metrics on the (group) manifold of unitaries. In this context, complexity becomes the length of the shortest geodesic between $U(0)=1$ and $U(\tau)$.

More precisely, we can decompose any circuit into infinitesimal gates $U_{H(\tau)} \equiv e^{-i H(\tau) d \tau}$, with $H(\tau)$ living in the tangent space at point $\tau$. In differential geometry, metrics are norms on the tangent space, so the most general Nielsen's cost is given by

$$
\mathcal{C}(\tau)=\int^{\tau} \mathcal{F}\left[H\left(\tau^{\prime}\right)\right] d \tau^{\prime},
$$

where $\mathcal{F}$ is some norm on the tangent space. In general, it is useful to think about such cost actions as describing a particle on a group manifold (e.g., sigma model type action given by length or energy), with associated equations of motion given by the geodesic equation.

Before defining $\mathcal{F}$, let us expand on the protagonist of this whole story, which is $H(\tau)$. We will call $H(\tau)$ "the instantaneous gate," since it takes us from $U(\tau)$ to $U(\tau+d \tau)$

$$
U(\tau+d \tau)=e^{-i H(\tau) d \tau} U(\tau) .
$$

Such instantaneous gates are the "velocities" in the group manifold. In general, their actual computation involves infinite sums of nested commutators (see, e.g., Ref. [30]), and it is impossible to write a closed form suitable for computing complexity. To make progress, in Ref. [27] one of us proposed to study submanifolds associated to symmetry groups $G$. In this scenario, gates are unitary representations $U_{g}$ of group elements $g$, and continuous protocols $U_{g(\tau)}$ are defined by paths $g(\tau)$ in the group. The instantaneous gate equation (3) has a homologous group equation

$$
g(\tau+d \tau)=e^{Q(\tau) d \tau} \cdot g(\tau)
$$

where $g \cdot g^{\prime}$ denotes the group product. The instantaneous gate is thus a Lie algebra element $-i H(\tau)=Q(\tau)$ (in the appropriate representation). For the mathematically oriented readers, $Q(\tau)$ is the adjoint transformation of the Maurer-Cartan form, known for many Lie groups, including the Virasoro group.

Next, we need to define the cost function $\mathcal{F}$ which is nonunique. Nielsen's proposal [1], in the context of spin systems, concerns two canonical choices, namely the oneand two-norms $\mathcal{F}_{1}$ and $\mathcal{F}_{2}$, which can be defined as the first and second moments of the infinitesimal gate in the maximally mixed state. This definition seems problematic in the case of continuous systems but, as discussed in Ref. [27], for symmetry gates this can be circumvented by replacing the "maximally mixed state" with the actual density matrix

$$
\rho(\tau) \equiv U(\tau) \rho_{0} U^{\dagger}(\tau)
$$

for some reference state $\rho_{0}$ that can be pure or mixed. Then, we define the norms as expectation values of the instantaneous symmetry gate(s) $Q(\tau)$ as

$$
\begin{aligned}
& \mathcal{F}_{1}(\tau) \equiv|\operatorname{Tr}[\rho(\tau) Q(\tau)]|=\left|\operatorname{Tr}\left[\rho_{0} \tilde{Q}(\tau)\right]\right|, \\
& \mathcal{F}_{2}(\tau) \equiv \sqrt{-\operatorname{Tr}\left[\rho(\tau) Q(\tau)^{2}\right]}=\sqrt{-\operatorname{Tr}\left[\rho_{0} \tilde{Q}(\tau)^{2}\right]},
\end{aligned}
$$

where both $Q(\tau)$ and $\tilde{Q}(\tau) \equiv U^{\dagger}(\tau) Q(\tau) U(\tau)$ are assumed to be given in the appropriate representation $U_{g}$. We remark that such costs are well defined from spin systems to interacting quantum field theories. Moreover, they are now physical quantities, the actual moments of the instantaneous rate of change of the state.

Finally, the complexity action (the length) is computed by Eq. (2) with either $\mathcal{F}_{1}$ or $\mathcal{F}_{2}$. As we will see below, this construction naturally fits into the framework of CFTs.

Circuit complexity in $2 D$ CFTs. - Now we consider the geometric approach to complexity in arguably the simplest setup relevant for holography, namely 2D CFTs (see Ref. [31] for references). We consider computational tasks in the (symmetry) unitary manifold that is at the core of every CFT, namely that of the Virasoro group. 
The Virasoro group is the central extension of the group of diffeomorphisms of the circle $\operatorname{Diff}^{+}\left(S^{1}\right)$ preserving orientation. Group elements are maps $f(\sigma)$ that are $2 \pi$ periodic $f(\sigma+2 \pi)=f(\sigma)+2 \pi$ and invertible $f^{\prime}(\sigma)>0$, as well as their group product is given by the composition

$$
f \cdot g=f \circ g .
$$

In 2D CFT, we have two copies of the Virasoro group that appear as the transformations of the cylinder light-cone coordinates. These diffeomorphisms are represented in the Hilbert space by unitary operators $U_{f}$, yielding, e.g., the well-known transformation of the stress tensor with Schwarzian derivative (see, e.g., Ref. [31]). Since our arguments are purely group theoretic, we first focus on a single copy.

To be precise, Virasoro elements are pairs $(f, \alpha)$ where $f \in \operatorname{Diff}^{+}\left(S^{1}\right)$ and $\alpha \in \mathbb{R}$. The product is the composition of functions and addition of numbers with the Bott cocycle (see Refs. [32,33] for pedagogical review). In the complexity context, we will not care about these numerical phases or, in other words, we associate cost zero to the identity gate.

Following the previous sections, we consider a unitary CFT circuit build from the Virasoro symmetry gates $U_{f}$ (unitary representations of the Virasoro group). Our Virasoro circuit $U(\tau)$ defines a path $f(\tau, \sigma)$ in the group manifold, taking us from a reference state $\left|\psi_{R}\right\rangle$ for $f(0, \sigma)=\sigma$ to a target state $\left|\psi_{T}\right\rangle=U_{f(\tau, \sigma)}\left|\psi_{R}\right\rangle$ for $f(\tau, \sigma)$ (to be clear: label $\tau$ means that for each $\tau$ we have one diffeomorphism $f_{\tau}(\sigma) \equiv f(\tau, \sigma)$ ).

The circuit is defined as

$$
U(\tau)=\overleftarrow{\mathcal{P}} \exp \left(\int_{0}^{\tau} Q\left(\tau^{\prime}\right) d \tau^{\prime}\right)
$$

where the instantaneous gate, that belongs to the Virasoro Lie algebra, can be expressed in terms of the stress tensor (see the Supplemental Material [34] for our conventions)

$Q(\tau) \equiv \int_{0}^{2 \pi} \frac{d \sigma}{2 \pi} \epsilon(\tau, \sigma) T(\sigma)=\sum_{n \in \mathbb{Z}} \epsilon_{n}(\tau)\left(L_{-n}-\frac{c}{24} \delta_{n, 0}\right)$,

where we Fourier expanded $\epsilon(\tau, \sigma)$ as well as the stress tensor on the cylinder in terms of the Virasoro algebra generators

$$
\left[L_{m}, L_{n}\right]=(m-n) L_{m+n}+\frac{c}{12} m\left(m^{2}-1\right) \delta_{m+n, 0} .
$$

The unitarity of our circuit is guaranteed by the condition $\epsilon_{n}^{*}(\tau)=-\epsilon_{-n}(\tau)$. However, as described in the Supplemental Material [34], this condition can be extended so that our circuits give the most general protocols in the CFT vacuum sector.

To compute the cost function, we need to relate $\epsilon(\tau, \sigma)$ (velocities) to the path $f(\tau, \sigma)$ itself. This is where the symmetry becomes crucial. Namely, since the group product is given by composition, by definition of the infinitesimal gate (4), we must have $\epsilon[\tau, f(\tau, \sigma)]=$ $\partial_{\tau} f(\tau, \sigma)$ or equivalently

$$
\epsilon(\tau, \sigma)=\partial_{\tau} f(\tau, F(\tau, \sigma))=-\frac{\partial_{\tau} F(\tau, \sigma)}{\partial_{\sigma} F(\tau, \sigma)},
$$

where we introduced the inverse function $F[\tau, f(\tau, \sigma)]=\sigma$. This explicit form of velocities allows us to express complexity as a functional of the path $f(\tau, \sigma)$. From now on we will denote $\partial_{\tau} f=\dot{f}$ and $\partial_{\sigma} f=f^{\prime}$ (and similarly for $F$ ).

As a reference state $\rho_{0}$ we will take a pure eigenstate of the CFT, created by a primary operator with (chiral) dimension $h: \rho_{0}=|h\rangle\langle h|$. Since a CFT basis can be obtained by acting with strings of $L_{n}$ 's on $|h\rangle$, we find this a natural choice to start with. Employing the Virasoro algebra (10) and using the transformation law of the stress tensor, we compute the one norm

$$
\mathcal{F}_{1}(\tau)=\frac{c}{24 \pi} \int_{0}^{2 \pi} d \sigma \frac{\dot{f}}{f^{\prime}}\left(2 a^{2}+\{f, \sigma\}\right),
$$

where $2 a^{2}=\frac{1}{2}[1-(24 h / c)]$. Similarly, we can evaluate the two norm (see the Supplemental Material [34] for the exact result)

$$
\mathcal{F}_{2}(\tau)=\mathcal{F}_{1}(\tau)[1+\mathcal{O}(1 / c)]
$$

We see that the two choices become equivalent in the large- $c$ limit, giving hope that some of the nonuniversal ambiguities of circuit complexity are "washed out" in holographic CFTs. Note also that the one-norm choice can be interpreted as the norm of the Berry connection for the Virasoro group.

This way, our large- $c$ complexity functional for paths $f(\tau, \sigma)$ between the identity and a given final transformation $f(\tau, \sigma)=f(\sigma)$ becomes

$$
\mathcal{C}[f](\tau)=\frac{c}{24 \pi} \int_{0}^{\tau} d \tau^{\prime} \int_{0}^{2 \pi} d \sigma \frac{\dot{f}}{f^{\prime}}\left(2 a^{2}+\{f, \sigma\}\right) .
$$

A simple but meaningful optimal protocol appears for $S L(2, R)$ paths with vanishing Schwarzian derivative. Then for the class of paths where $\dot{f} / f^{\prime}=c_{1}$ is constant, we arrive to the linear complexity growth [35]

$$
\mathcal{C}[f](\tau)=c_{1} E_{h} \tau,
$$


where $E_{h}=|h-(c / 24)|$ is the expectation value of the energy-momentum tensor. More complicated examples will be explored in Ref. [36].

Finally, performing analogous steps for the second copy of the Virasoro group, the full CFT complexity action reads

$$
\mathcal{C}^{\mathrm{CFT}}[f, \bar{f}](\tau)=\mathcal{C}[f](\tau)+\mathcal{C}[\bar{f}](\tau) .
$$

where $\bar{f}$ is the analogous (independent) conformal path for the left Virasoro group. Above we have used $Q=$ $Q_{L}+Q_{R}$. Had we defined a cost mediated by a negative sign $Q=Q_{L}-Q_{R}$, we would have obtained $\mathcal{C}[f]-\mathcal{C}[\bar{f}]$. At the level of the classical solutions, there is no difference between both choices.

We remark again that the cost is written in terms of Lie algebra elements. In AdS/CFT, these are boundary operators, with known dual fields. Therefore, our costs are built from well-defined quantities at both sides of the AdS/CFT duality. In fact, we will now discuss a more direct connection between our complexity and gravity.

$2 D$ gravity.-The connection of the 2D-CFT complexity functional (14) to gravity appears via the Polyakov's action of (induced) gravity in two dimensions [28]

$$
S_{P}[g]=\frac{c}{24 \pi} \int d^{2} x \sqrt{g}\left(-\frac{1}{4} R \frac{1}{\square} R+\Lambda\right),
$$

where $R$ is the Ricci scalar curvature, $\Lambda$ the cosmological constant, and $\square$ the Laplace-Beltrami operator (its inverse $1 / \square$ acting on $R$ is just a formal expression implying that $R \equiv \square(\ldots))$. Polyakov action is intimately related to Virasoro symmetry, and it plays the role of the generating functional of the stress tensor correlators

$$
e^{-S_{P}[\mu]} \equiv\left\langle e^{-\frac{1}{2 \pi} \int d \tau d \sigma \mu \mathbf{T}}\right\rangle,
$$

where $S_{P}[\mu]$ is the Polyakov action computed in metric (Polyakov gauge) [28]

$$
d s^{2}=d \tau(d \tilde{\sigma}+\mu(\tau, \tilde{\sigma}) d \tau)=G^{\prime}(\tau, \sigma) d \tau d \sigma,
$$

where $\mu=\dot{g} / g^{\prime}$ and $g[\tau, G(\tau, \sigma)]=\sigma$.

This way, we have, e.g., the one-point function

$$
\frac{\delta}{\delta \mu} S_{P}[\mu]=\frac{1}{2 \pi}\langle\mathbf{T}\rangle=\frac{c}{24 \pi}\{g(\tau, \sigma), \sigma\} .
$$

For our purposes, the large- $c$ limit ensures that $\left\langle e^{-(1 / 2 \pi) \int d \tau d \sigma \mu \mathbf{T}}\right\rangle \simeq e^{-(1 / 2 \pi) \int d \tau d \sigma \mu\langle\mathbf{T}\rangle}$. Hence, our complexity becomes the Polyakov action of induced 2D gravity. This is the main result of our work and a completely new light on this well-established core of 2D CFTs.

Let us elaborate more on this important connection and bring a new light that allows for generalizations toward complexity in quantum field theories.
The Polyakov action evaluated on metric (19) can be compactly written as

$$
S_{P}[G]=\frac{c}{24 \pi} \int d \tau \int d \sigma \frac{\dot{G}}{2 G^{\prime}}\left(\frac{G^{\prime \prime \prime}}{G^{\prime}}-2 \frac{G^{\prime \prime 2}}{G^{\prime 2}}\right),
$$

and, as proven in Refs. [37,38], it leads to the same e.o.m and expectation value of $\mathbf{T}$ as the one with full Schwarzian derivative $S_{P}[\mu] \sim \int \mu\{g, \sigma\}$. Were we on the plane, this would be our large- $c$ complexity functional. As shown in Refs. $[39,40]$, on the cylinder we just need to substitute $G=\exp (\sqrt{2} a F)$ and using $f[\tau, F(\tau, \sigma)]=\sigma$ one arrives to Ref. (14). This makes the precise connection with the functions $F$ and $f$ (and parameter $a$ ) used in our complexity functional.

Let us point that for two Virasoro copies we get two Polyakov or complexity actions. It is well known $[41,42]$ how they can be combined into a single, nonchiral Liouville theory. This sheds new light on the path integral complexity proposal [15]. It is an important future problem to compare the details of the two approaches [36] (see also Refs. [43-45] for related constructions).

Finally, as shown in Ref. [29], Polyakov action (21) is in fact the Kirillov geometric action [30] on the coadjoint orbits of the Virasoro group (see the Supplemental Material [34]). More precisely, the Kirillov geometric method was applied to the Virasoro group in Refs. [29,32] and the resulting geometric action is given by

$$
I_{\text {Virasoro }}=\frac{c}{24 \pi} \int d \tau d \sigma \frac{\dot{f}}{f^{\prime}}\left[\frac{12}{c} b(f)+\frac{1}{2}\left(\frac{f^{\prime \prime}}{f^{\prime}}\right)^{\prime}\right] .
$$

The $b(f)$ is an element of the dual space of the Virasoro Lie algebra and labels the specific coadjoint orbit (function $f$ is the same as above: $f[\tau, F(\tau, \sigma)]=\sigma)$. We can identify it with the stress tensor expectation value $b(f)=\operatorname{Tr}\left[\rho_{0} T(f)\right]$ and in our case of the primary state $b(f)=h-(c / 24)=$ $-(c / 6) a^{2}$ (in general the dependance on $\sigma$ just means that $b(f)$ transforms as the expectation value of $T(\sigma))$. The second contribution is the first term of the Schwarzian derivative and, as functional for $f$, this action is equivalent to Ref. (14). Aspects of the above geometric action were also used recently in connection to SYK [46-48] and Virasoro Berry phases [49].

As we will discuss in the last section, the fact that we can write our complexity as a geometric action gives a hint and a definite route for constructing complexity (based on "generalized symmetry gates") in arbitrary quantum field theories.

Generalizations and discussion.-The most promising generalization relies on the observed connection between complexity and geometric actions. Such geometric actions appear ubiquitously through the notion of generalized coherent states; see Refs. [50-53]. Basically, for any quantum theory there exists a "coherence group" $G$. 
This group provides a classical phase space for the theory, by generating a continuous and normalizable Hilbert space basis, the coherent states $\left|g_{v}\right\rangle=U(g)|v\rangle$. In the semiclassical limit, the dynamic is determined by an action

$$
S=S_{\text {geometric }}+S_{\mathrm{H}}=-\int_{\gamma} \alpha-\int d \tau H[\gamma(\tau)],
$$

where $\gamma(\tau)$ is a path on the coherence group and $H[\gamma(\tau)]$ is the Hamiltonian (a functional on phase space). In this context, natural gates are infinitesimal elements of the coherence group, states are elements of the dual space, and protocols are paths $\gamma(\tau) \in G$ defining paths in phase space by means of the coadjoint transformation. Using the same notion of cost (6), in the semiclassical limit the complexity is again given by the geometric action

$$
\mathcal{C}=\int_{\gamma} \alpha
$$

By comparing the semiclassical solution (23) and the complexity or geometric action (24), the analysis suggests that for theories controlled by a Hamiltonian constraint $H[\gamma(\tau)]=0$ the complexity action is exactly the classical action. This might connect nicely to gravitational theories.

In AdS/CFT, this coherent state approach can be defined on both sides of the duality, since instantaneous gates are smeared versions of local boundary operators, with known gravity duals, and associated Kirillov-Kostant symplectic forms are functionals of them (see, e.g., Refs. [27,54]). An important subset of the coherence group is $e^{i \int f(x) g(x)}$, with $g$ the metric, dual to $e^{i \int f(x) T(x)}$, with $T$ the stress tensor. In $2 \mathrm{D}$, this generates the conformal group and has been considered above.

Related generalizations within 2D CFTs arise when allowing instantaneous gates to include other primaries or symmetry currents (like Kac-Moody or higher spin W), or when including supersymmetry. Indeed, geometric actions for the Kac-Moody symmetry are well known and the approach of Alexeev and Shatashvili was also generalized to $W_{3}$ (see, e.g., Refs. $[55,56]$ ).

We conclude that the present approach provides a novel starting point to derive gravity from complexity. In our setup, the CFT complexity corresponds to the Virasoro geometric action for each left and right sector. References [29,57] showed how each chiral sector is equivalent to a $S L(2, \mathbf{R})$ Wess-Zumino-Witten (WZW) theory. In turn, these WZW theories are the reduction at the boundary of $\mathrm{AdS}_{3}$ of the two Chern-Simons actions of 3D gravity [58]. Moreover, solutions of 3D gravity are fixed by solutions of the 2D Liouville equation, parametrized by our $\operatorname{Diff}\left(S^{1}\right)$ diffeomorphisms. Indeed, from a nonchiral Liouville field, we can construct left and right stress tensors completely specifying a three-dimensional metric [59].
Also, the alluded Liouville action computes the area of a two-dimensional surface, naturally associated with a slice of a dual bulk geometry (see Ref. [15] for Euclidean slices). Even though it is by no means clear which slice this may be, we conjecture that it is always possible to find such a slice in the bulk.

We wish to thank Hugo Camargo, Horacio Casini, Sumit Das, Jan de Boer, Nilay Kundu, Hugo Marrochio, Gautam Mandal, Rob Myers, Masamichi Miyaji, Tokiro Numasawa, Blagoje Oblak, Onkar Parrikar, Fernando Pastawski, Massimo Poratti, Guifre Vidal, and especially Joan Simon and Tadashi Takayanagi for guidance and important suggestions. Our work is supported by the Simons Foundation through the "It from Qubit" collaboration and work of P. C. was also supported by the JSPS starting Grant No. KAKENHI 17H06787. We thank the Galileo Galilei Institute for Theoretical Physics for the hospitality and the INFN for partial support during the completion of this work.

[1] M. A. Nielsen, arXiv:quant-ph/0502070.

[2] M. A. Nielsen, M. R. Dowling, M. Gu, and A. C. Doherty, Science 311, 1133 (2006).

[3] M. R. Dowling and M. A. Nielsen, arXiv:quant-ph/ 0701004

[4] J. M. Maldacena, Adv. Theor. Math. Phys. 2, 231 (1998); Int. J. Theor. Phys. 38, 1113 (1999).

[5] L. Susskind, Fortschr. Phys. 64, 44 (2016).

[6] D. Stanford and L. Susskind, Phys. Rev. D 90, 126007 (2014).

[7] A. Brown, D. Roberts, L. Susskind, B Swingle, and Y. Zhao, Phys. Rev. Lett. 116, 191301 (2016).

[8] A. Brown, D. Roberts, L. Susskind, B Swingle, and Y. Zhao, Phys. Rev. D 93, 086006 (2016).

[9] S. Aaronson, arXiv:1607.05256.

[10] A. Brown and L. Susskind, Phys. Rev. D 97 (2018) 086015.

[11] M. Miyaji, T. Numasawa, N. Shiba, T. Takayanagi, and K. Watanabe, Phys. Rev. Lett. 115, 261602 (2015).

[12] M. Alishahiha, Phys. Rev. D 92, 126009 (2015).

[13] S. Chapman, H. Marrochio, and R. C. Myers, J. High Energy Phys. 01 (2017) 062.

[14] L. Lehner, R. C. Myers, E. Poisson, and R. D. Sorkin, Phys. Rev. D 94, 084046 (2016).

[15] P. Caputa, N. Kundu, M. Miyaji, T. Takayanagi, and K. Watanabe, Phys. Rev. Lett. 119, 071602 (2017); J. High Energy Phys. 11 (2017) 097.

[16] B. Czech, Phys. Rev. Lett. 120, 031601 (2018).

[17] R. Jefferson and R. Myers, J. High Energy Phys. 10 (2017) 107.

[18] S. Chapman, M. Heller, H. Marrochio, and F. Pastawski, Phys. Rev. Lett. 120, 121602 (2018).

[19] R. Yang, Phys. Rev. D 97, 066004 (2018).

[20] K. Kim, C. Niu, R. Yang, and C. Zhang, J. High Energy Phys. 02 (2018) 082.

[21] R. Khan, C. Krishnan, and S. Sharma, Phys. Rev. D 98, 126001 (2018). 
[22] K. Hashimoto, N. Iizuka, and S. Sugishita, Phys. Rev. D 96, 126001 (2017); Phys. Rev. D 98, 046002 (2018).

[23] J. Molina-Vilaplana and A. Del Campo, J. High Energy Phys. 08 (2018) 012.

[24] R. Abt, J. Erdmenger, H. Hinrichsen, C. M. MelbyThompson, R. Meyer, C. Northe, and I. A. Reyes, Fortschr. Phys. 66, 1800034 (2018).

[25] L. Hackl and R. C. Myers, J. High Energy Phys. 07 (2018) 139.

[26] M. Flory and N. Miekley, J. High Energy Phys. 05 (2019) 003.

[27] J. Magan, J. High Energy Phys. 09 (2018) 043.

[28] A. M. Polyakov, Mod. Phys. Lett. A 02, 893 (1987).

[29] A. Alekseev and S. L. Shatashvili, Nucl. Phys. B323, 719 (1989); Rev. Math. Phys. 30, 1840001 (2018).

[30] A. A. Kirillov, Lectures on the Orbit Method, Graduate Studies in Mathematics (American Mathematical Society, Providence, 2004).

[31] P. Di Francesco, P. Mathieu, and D. Senechal, Conformal Field Theory (Springer, New York, 1997).

[32] E. Witten, Commun. Math. Phys. 114, 1 (1988).

[33] B. Oblak, arXiv:1610.08526.

[34] See Supplemental Material at http://link.aps.org/ supplemental/10.1103/PhysRevLett.122.231302 for a review of our conventions, more detailed computations, different choices of the complexity metric (including Fubiny-Study and Euler-Arnold) and a review of so-called geometric actions in group theory.

[35] S. Lloyd, Nature (London) 406, 1047 (2000).

[36] P. Caputa and J. Magan (to be published).

[37] E. Aldrovandi and L. A. Takhtajan, Commun. Math. Phys. 188, 29 (1997).

[38] Z. Haba, Phys. Rev. D 41, 724 (1990).
[39] A. Alekseev and S. L. Shatashvili, Commun. Math. Phys. 128, 197 (1990).

[40] G. Barnich, H. A. Gonzalez, and P. Salgado-Rebolledo, Classical Quantum Gravity 35, 014003 (2018).

[41] M. Henneaux, L. Maoz, and A. Schwimmer, Ann. Phys. (N.Y.) 282, 31 (2000).

[42] O. Coussaert, M. Henneaux, and P. van Driel, Classical Quantum Gravity 12, 2961 (1995).

[43] A. Milsted and G. Vidal, arXiv:1805.12524.

[44] A. Milsted and G. Vidal, arXiv:1807.02501.

[45] J. Molina-Vilaplana, Phys. Lett. B 755, 421 (2016).

[46] D. Stanford and E. Witten, J. High Energy Phys. 10 (2017) 008.

[47] G. Mandal, P. Nayak, and S. R. Wadia, J. High Energy Phys. 11 (2017) 046.

[48] T. G. Mertens, J. High Energy Phys. 05 (2018) 036.

[49] B. Oblak, J. High Energy Phys. 10 (2017) 114.

[50] J. R. Klauder and E. C. G. Sudarshan, Fundamentals of Quantum Optics (Benjamin, New York, 1968).

[51] A. M. Perelomov, Commun. Math. Phys. 26, 222 (1972).

[52] L. G. Yaffe, Rev. Mod. Phys. 54, 407 (1982).

[53] J. M. Magan, Phys. Rev. D 96, 086002 (2017).

[54] A. Belin, A. Lewkowycz, and G. Sarosi, Phys. Lett. B 789, 71 (2019).

[55] A. Marshakov and A. Morozov, Nucl. Phys. B339, 79 (1990); Sov. Phys. JETP 70, 403 (1990).

[56] H. Ooguri, K. Schoutens, A. Sevrin, and P. van Nieuwenhuizen, Commun. Math. Phys. 145, 515 (1992).

[57] P. Forgacs, A. Wipf, J. Balog, L. Feher, and L. O'Raifeartaigh, Phys. Lett. B 227, 214 (1989).

[58] E. Witten, Nucl. Phys. B311, 46 (1988).

[59] M. Banados, AIP Conf. Proc. 484, 147 (1999). 